\title{
Temperature-Modulated Calorimetry of Polymers with Single and Multiple Frequencies to Determine Heat Capacities as well as Reversible and Irreversible Transition Parameters *
}

Reference: Wunderlich, B., "Temperature-Modulated Calorimetry of Polymers with Single and Multiple Frequencies to Determine Heat Capacities as well as Reversible and Irreversible Transition Parameters," Material Characterization by Dynamic and Modulated Thermal Analytical Techniques, ASTM STP 1402, A. Riga and L. Judovits, Eds., American Society Testing and Materials, Conshohocken, PA, 2001.

Abstract: Temperature-modulated differential scanning calorimetry (TMDSC) generated with a centrosymmetric saw-tooth oscillation can be considered to be a sinusoidal modulation with multiple frequencies. Different harmonics of the Fourier series of the heat-flow rate and heating rate of a single sawtooth-modulation can be deconvoluted to extract data pertaining to different frequencies. In order to give the higher harmonics similar amplitudes, a complex, but simple-to-program, sawtooth-modulation is generated for the harmonics $1,3,5,7$ and 9 . In this fashion a single experiment can produce a frequency-dependent analysis under identical thermal history. Application of this method to TMDSC includes the calibration for heat capacity determination of high precision, even if steady state and a negligible temperature gradient are not achieved. The measurement of the frequency $(\omega)$ dependence of the heatflow rate $\left(A_{\mathrm{HF}}\right)$ and sample temperature $\left(A_{\mathrm{T}_{\mathrm{s}}}\right)$ allows to evaluate the expression:

$$
C_{\mathrm{p}}=A_{\mathrm{HF}} /\left(A_{\mathrm{T}_{\mathrm{s}}} \nu \omega\right)\left[1+(\tau \vee \omega)^{2}\right]^{0.5} \text {, }
$$

where the relaxation time $\tau$ is to be determined empirically from the multiple data generated by the single run. Typical values for the relaxation time for commercial calorimeters are between 3 and $9 \mathrm{~s} \mathrm{rad}^{-1}$. Frequency-dependent, apparent reversing heat capacities in the glass transition region and within first-order transition regionss may also be analyzed to study local equilibria in globally metastable polymeric solids.

Keywrords: temperature-modulated DSC, multifrequency modulation, metastable polymer, heat capacity, latent heat, phase transition, glass transition, first-order transition

1 Department of Chemistry, The University of Tennessee, Knoxville, TN 37996-1600, and the Chemistry and Analytical Sciences Division at Oak Ridge National Laboratory, Oak Ridge, IN 37831-6197, USA.

\footnotetext{
"This submitted manuscript has been authored by a contractor of the U.S. Government under the contract No. DOE-AC05-00OR22725. Accordingly, the U.S. Government retains a nonexclusive, royalty-free license to publish Or reproduce the published form of this contribution, or allow others to do so, for U.S. Government purposes."
} 


\section{Introduction}

Temperature-modulated differential scanning calorimetry (TMDSC) had its beginning in 1992 with the introduction of the first commercial calorimeter by TA Instruments, Inc. [ 1 ]. The special feature of the TMDSC is in the simultaneous evaluation of the thermal effect due to a slow, constant-rate temperature increase (typically 0.1 to $5 \mathrm{~K} \mathrm{~min}^{-1}$ ), the "total differential heat-flow rate," similar to the output of a standard, not modulated DSC, and a faster effect, the response to the temperature modulation, called the "reversing differential heat-flow rate" (typical temperature modulation parameters are an amplitude of 0.05 to $3 \mathrm{~K}$ and a period from 10 to $500 \mathrm{~s}$ ). Naturally the differences between these two quantities are then the "non-reversing differential heat-flow rates." Since only the combined signal is generated, a deconvolution procedure is necessary, usually carried out in form of a discrete Fourier analysis of the sample temperature $T_{s}(t)$ and the heat-flow response $H F(t)$.

The root of measurements with modulation of temperature is the alternating current, ac calorimeter [2]. Some attempts were also made at the development of twin ac calorimeters [3]. In all cases, however, the ac calorimeters were run quasi-isothermally, i.e., the modulation occurred about a constant temperature, $T_{0}$, yielding only the reversing response to the modulation. In this paper the special problems and advantages of TMDSC are traced as they became evident from work on multifrequency modulation in our laboratory [4]. Multifrequency modulation was already proposed in the TMDSC patent [1], but not studied.

\section{Standard DSC with Negligible Temperature Gradient and at Steady State}

The standard, not-modulated DSC can easily be described as long as one assumes a negligible temperature gradient within the sample and steady state during the period of measurement [5]. Under such idealized conditions, the heat flows of the sample calorimeter, consisting of pan and sample, and the reference calorimeter, usually only an empty pan, are governed solely by the heating rate, $q$, and their heat capacities, written as $C_{s}=\left(m c_{p}+C_{p}{ }^{\prime}\right)$ and $C_{\mathrm{r}}=C_{\mathrm{p}}{ }^{\prime}$, respectively, as is shown in Figure 1. The heat flows fenerated by the different

\section{Typical Standard DSC}

\section{(TA Instruments DSC 910)}

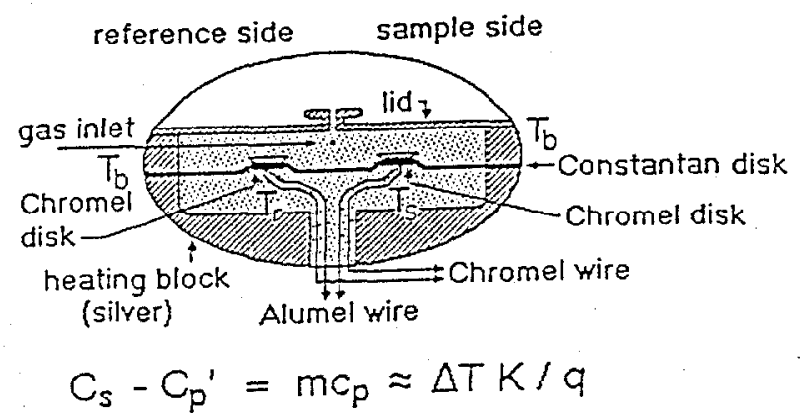

\footnotetext{
$C_{s}=$ sample calorimeter $C_{p}$

$m=$ sample mass

$c_{p}=$ sample specific heal capacity

$C_{p}=$ pan heat capacity
}

$\mathrm{q}=$ heating rate

$\begin{aligned} q & =\text { healing } \\ \Delta T & =T_{r}-T_{s}\end{aligned}$

$K=$ Newton's law constant

$\mathrm{C}_{r}=$ reference calorimeter $\mathrm{C}_{p}$

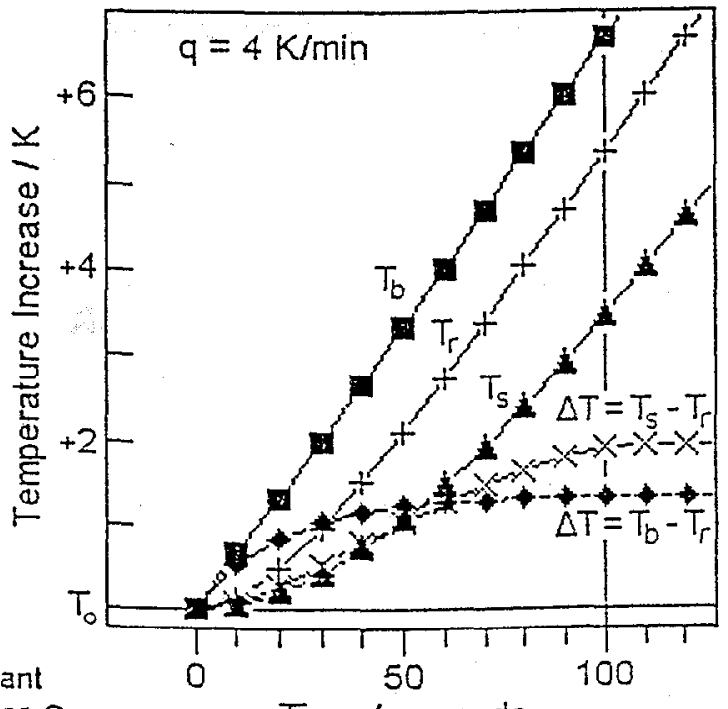

Time / seconds

Figure 1 - Schematics and operation of a standard DSC. 
temperatures are proportional to the temperature differences $\Delta T$. The experiment is started at time zero with a linear temperature increase of the heater, $T_{b}$. After about $120 \mathrm{~s}$, the reference and sample temperatures reach steady state, i.e., both increase with the same $q$ as $T_{b}$, and $\Delta T$ becomes constant. The data in Figure 1 are calculated by assuming Newton's law constant, $K$, to be $\mathrm{C}_{\mathrm{p}} / 20 \mathrm{~J} \mathrm{~K}^{-1} \mathrm{~s}^{-1}$, and that the Fourier equation of heat flow is valid [5].

A minor correction of the equation shown in Figure 1 is needed since the sample and reference calorimeters change their heat capacities with temperature, i.e., the $\Delta T$-curves in Figure 1 are not strictly parallel to $T_{b}$. This correction needs no further measurement and could be derived some time ago [ 0 ] using equations given by Ozawa [ $]$ :

$$
m c_{\mathrm{p}}=K \frac{\Delta T}{q}+C_{\mathrm{s}}\left(\frac{\mathrm{d} \Delta T}{\mathrm{~d} T_{\mathrm{s}}}\right) .
$$

All of the equation symbols are explained in Figure 1. The correction is typically the order of magnitude of $1 \%$ as long as the $m c_{p}$ is a substantial portion of $C_{s}$. Similarly, the negligible temperature gradient within the sample is not a stringent condition as long as steady state is kept. A temperature gradient of $2.0 \mathrm{~K}$ across a sample of crystalline polyethylene will cause an error in the measurement of the magnitude of $d c_{p} / d T$, or about $0.3 \%$ at $300 \mathrm{~K}[8]$.

\section{Sinusoidal and Saw-tooth Modulation}

The simplicity of the data analysis for a standard DSC is lost, as soon as temperature modulation is added. In particular, depending on the modulation method, different results are expected. In Figure 2, the curves on the left illustrate a sinusoidal modulation, and the ones on the right, a quasi-isothermal, sawtooth modulation. For simplicity, in both cases the heater temperature is modulated. In a quasi-isothermal experiment, the average rate of temperature change, $\langle q\rangle$, is zero. The input parameters for the calculation on the right are the same as used for Figure 1, just that at time $t_{1}=100 \mathrm{~s}$ the heating rate is changed into cooling. The equations derived for the description of the curves on the right are listed in the figure. As long as the heat flows are described by the Fourier equation of heat flow, the solutions for
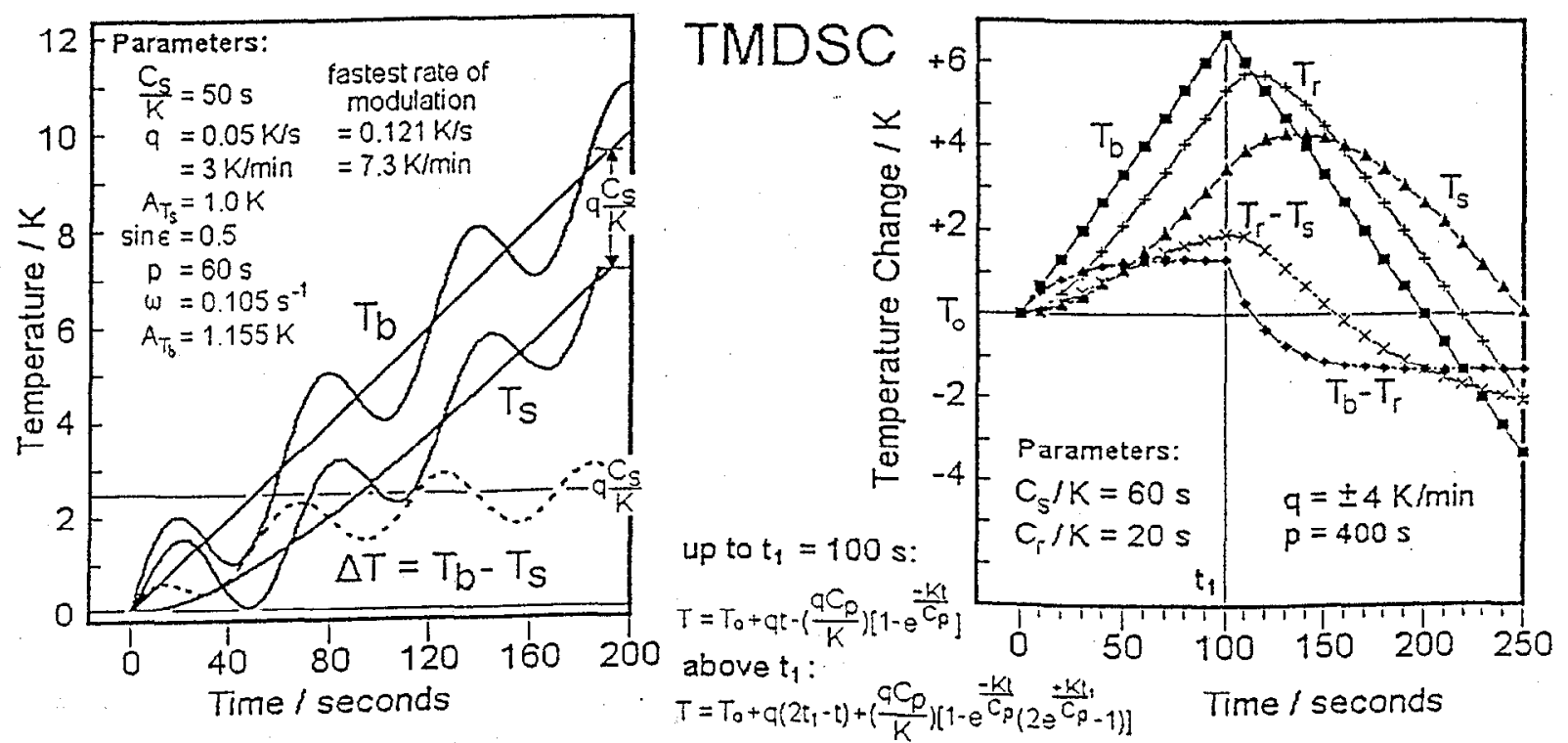

Figure 2 - Sinusoidal and sawtooth modulation. 
different events are additive. Beyond time $t_{1}$, for example, one can describe the temperature changes of $T_{\mathrm{s}}$ and $T_{\mathrm{r}}$ by assuming the heating is terminated at $t_{1}$, resulting in an approach to an isotherm at $+6.67 \mathrm{~K}$, and simultaneously a new experiment of cooling is initiated [5]. The sum of these two events yields the bottom equation in Figure 2 and is plotted in the curves beyond $t_{1}$. The top equation describes the temperatures $T_{s}$ and $T_{r}$ for times up to $t_{1}$.

An immediate observation is that the sinusoidal modulation reaches after a few cycles a constant average level, and that the sliding averages over one modulation period $\left\langle I_{b}\right\rangle$, $\left\langle T_{\mathrm{s}}\right\rangle$, and $\langle\Delta T\rangle$ yield the same curves as seen in Figure 1 for standard DSC. A simple subtraction of this average from the instantaneous values results in the contribution due to the modulation alone, the reversing signal. The signal applies pseudo-isothermally to $\langle T(t)\rangle$. The deconvolution of the two results of TMDSC is thus quite simple after about $200 \mathrm{~s}$.

An extremely easy analysis of the reversing heat capacity is possible as long as $\left\langle T_{b}\right\rangle$, $\left\langle T_{\mathrm{s}}\right\rangle$, and $\left\langle T_{\mathrm{r}}\right\rangle$ change linearly with time. At every time or temperature, $\left\langle T_{\mathrm{s}}(t)\right\rangle-T(t)$ is identical to that expected from a quasi-isothermal experiment under the same condition except that $\langle q\rangle \neq 0$. The quasi-isothermal analysis has been investigated in detail and yields for the heat capacity the following expression [9] which also holds for the pseudo-isothermal case:

$$
\left(C_{\mathrm{s}}-C_{\mathrm{r}}\right)=\frac{A_{\Delta} K}{A_{\mathrm{r}_{\mathrm{s}}} \omega} \sqrt{1+\left(\frac{C_{\mathrm{r}} \omega}{K}\right)^{2}},
$$

where $A_{\Delta}$ is the modulation amplitude of $\Delta T$ and $A_{\mathrm{T}}$, that of $T_{\mathrm{s}}$. The frequency $\omega$ is given in $\mathrm{rad} \mathrm{s} \mathrm{s}^{-1}$. The similarity of Equations (2) and (1) becomes obvious if one uses an empty reference calorimeter. Then, $C_{\mathrm{r}}$ is equal to $C^{\prime}$ and $C_{\mathrm{s}}-\mathrm{C}_{\mathrm{r}}=m c_{\mathrm{p}}$, and $A_{\mathrm{T}_{\mathrm{s}}} \omega$ represents the amplitude of the modulation of the heating rate $q(t)-\langle q\rangle=\mathrm{d} T_{s}(t) / \mathrm{d} t$. The square root accounts, as in Equation (1), for the difference between the modulation of reference and sample calorimeter. Only if there is an empty reference position (no pan, $C_{\mathrm{r}}=$ zero) does Equation (2) change into:

$$
c_{\mathrm{s}}=m c_{\mathrm{p}}+C^{\prime}=\frac{A_{\Delta} K}{A_{\mathrm{T}_{\mathrm{s}}} \omega},
$$

an equation often erroneously also used with a reference calorimeter $\left(C_{\mathrm{r}} \neq 0\right)$. Note, however, that if 'calibration and measurement are done at the same frequency and the reference pans do not change between runs, all differences between Equations (2) and (3) can be eliminated by calibration, $K$ of Equation (3) is then $K \times$ [the square root of Equation (2)].

The just discussed Equations (2) and (3) hold only if steady state is not lost during modulation and the temperature gradient within the calorimeters is negligible. This condition is more stringent than for the standard DSC, because if even a small temperature gradient is set up within the sample during the modulation, each modulation cycle has smaller positive and negative heat flows that depend on the unknown thermal conductivities. A negligible temperature gradient within the sample requires, thus, that the sample calorimeter oscillates in its entirety as shown in the left graph of Figure 2. It also requires a negligible thermal resistance between thermometer and pan, and the pan and sample calorimeter. The phase lag $\varepsilon$ between heater and sample must in this case be entirely due to the thermal diffusivity of the Constantan disk $\left[T_{s}(t)=A_{T_{s}} \sin (\omega t-\varepsilon)\right]$. Typical conditions that have been used for measuring $C_{\mathrm{p}}$ of polymers with sinusoidal modulation are masses of about $10 \mathrm{mg}$, amplitudes of modulation of $1.0 \mathrm{~K}$, and modulation periods of $60 \mathrm{~s}$ or longer. 


\section{Measurement Without Reaching Steady State and With Temperature Gradients}

Turning to the sawtooth modulation displayed on the right-hand side of Figure 2, steady state is lost at every sharp change of $q$, at $t_{1}$. In the chosen calculation condition, the heat-flow rate of the sawtooth is not in steady-state during about half the modulation period of $400 \mathrm{~s}$, i.e., there should be no way to measure heat capacities using Equations (2) or (3). Attempts were made to use the maxima and minima of the modulation response. This corresponds to a use of a standard DSC to test for the reversing nature of the sample and had been proposed some time ago (DDTA, dynamic differential thermal analysis [10]) Naturally this simple measurement is only correct if steady state is reached in each half cycle.

An extensive analysis of the sawtooth modulation with the Perkin Elmer calorimeter brought a number of interesting results. Mathematically it could be shown that if there were no temperature gradient within the sample and if all lags and gradients could be assessed with the Fourier heat-flow equation, Equation (2) does allow the calculation of the precise heat capacities [4]. Since, however, the temperature sensor of the power compensated calorimeter is much closer to the heater than the sample, temperature gradients cannot be avoided. The empirical solution to this problem was to modify Equation (3) as follows [11]:

$$
\left(C_{s}-C_{r}\right)=\frac{A_{\Delta} K}{A_{T_{s}} \omega} \sqrt{1+(\tau \omega)^{2}},
$$

where $\tau$, which has the dimension $\mathrm{s} \mathrm{rad}^{-1}$, is an adjustable constant to be determined by measurements at different frequencies. It depends not only on the heat capacity of the reference calorimeter and the Newton's-law constant, as one would expect from Equation (2), but also on the mass and thermal conductivity of the sample, as well as on all of the involved thermal contact resistances and possibly also on cross-flow between sample and reference calorimeters, depending on the calorimeter type. Modeling of such complicated situations has been attempted by Hatta [12], Höhne [13], and others, but it is difficult to evaluate the various constants such treatments generate. As a result, we decided to use Equation (4) as a tool to study $\tau$ empirically. As long as only sample mass and thermal conductivity are affecting $\tau$ (in addition to $C_{\mathrm{r}}$ and $K$ ), a plot of the inverse of the uncorrected heat capacity of Equation (3) versus the square of the frequency should be linear, as it was indeed found for the Perkin Elmer DSC with modulation periods longer than $15 \mathrm{~s}$ (quasi-isothermal, $T_{0}=298$ $\mathrm{K}$, with modulation amplitudes from one to four kelvins) [11]. Only with modulation periods beyond about $250 \mathrm{~s}$ did the frequency dependence become fully negligible. Before continuing this discussion, it is now necessary to briefly review the evaluation of the amplitudes of modulation and the heat-flow response and point out some interesting facts.

\section{Evaluation of the Fourier Components of the Sawtooth Modulation}

The sample temperature, $T_{s}(t)$ and the reversing heat-flow rate response, $H F(t)$ which is proportional to $\Delta T(t)$, are commonly represented by the amplitude of the first hamonic of the Fourier representations. For the amplitude of the heat-flow rate, $A_{\mathrm{HF}}$, one obtains:

$$
H F(t)=\langle H F\rangle+\sum_{v=1}^{\infty}\left[A_{v} \sin (\nu \omega t)+B_{v} \cos (\nu \omega t)\right]
$$

where $A_{v}$ and $B_{v}$ are coefficients that must be determined in the usual manner, and $v$ is an 


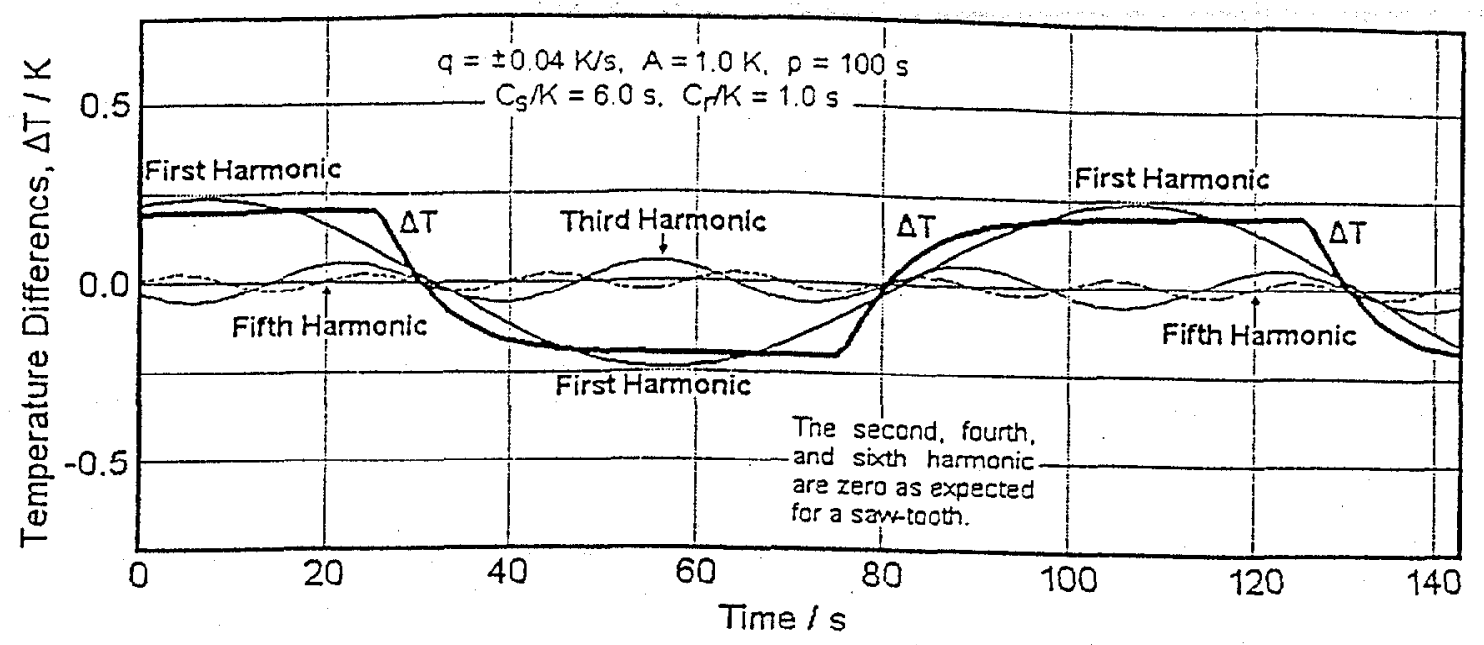

Figure 3 - Sawtooth model calculation for $\Delta T(t)$ and its harmonics.

integer. An analogous equation has to be used for the sample temperature. As long as the modulation starts at $t=0$ and is symmetric about $\left\langle q>t\right.$, it is centrosymmetric and all $B_{v}$ are zero, i.e., the series contains only the sinusoidal harmonics. For a linear response of the sample to a sinusoidal modulation, no higher harmonics are generated in the heat-flow rate, i.e., $A_{\mathrm{HF}}=A_{1}$ of Equation (5). A centrosymmetric sawtooth modulation also simplifies the Fourier representation, it shows only odd, sinusoidal harmonics with $v=1,3,5$, etc.

Figure 3 illustrates the temperature difference, $\Delta T(t)$, for a centrosymmetric sawtooth modulation indicating the influence of the higher harmonics for the case of a modulation that reaches steady state after about half of every heating and cooling segment. If Equation (2) describes the TMDSC, each sinusoidal harmonic can separately be used to compute the heat capacity. Although the amplitudes of the higher harmonics decrease quickly, up to the $11^{\text {th }}$ harmonics could be used to establish $\tau$. Figure 4 shows the results for a typical copolymer and sapphire, analyzed with a Perkin-Elmer calorimeter [14]. Several runs with different modulation periods were used and the uncorrected heat capacities as calculated from Equation (3) are plotted on the left, and the corrected values from Equation 4 are plotted on
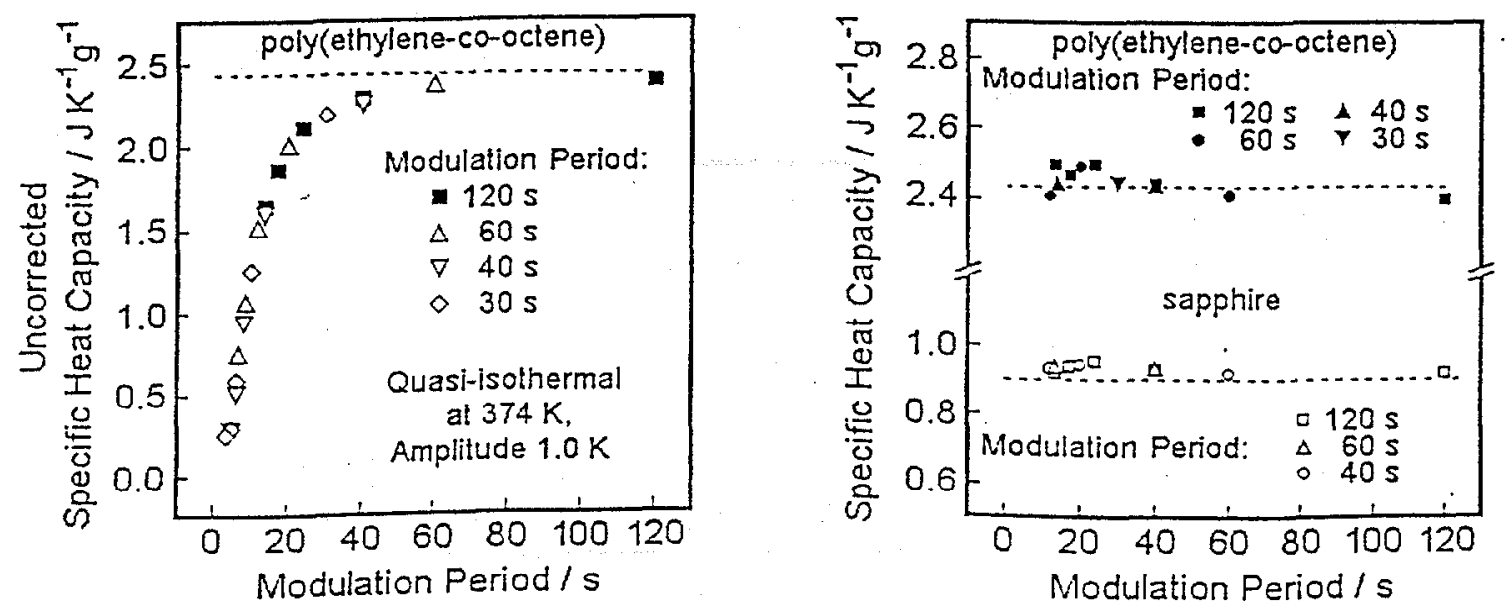

Figure 4-Analysis with Equations (3) and (4). 
the right for measurements that show a frequency-independent $\tau$ for higher harmonics with periods longer than $10-15 \mathrm{~s}\left(\tau_{\text {polymer }}=2.40 \mathrm{~s} \mathrm{rad}^{-1}\right.$ and $\left.\tau_{\text {sapphire }}=2.24 \mathrm{~s} \mathrm{rad}^{-1}\right)$. The dashed lines indicate the expected value of the heat capacity from the literature. Note that the calibration run with sapphire needs a different $\tau$, and can thus only be used after evaluation of its separate $\tau$ value and extrapolation to zero frequency. It could also be shown that the common practice of subtracting a baseline of a run with two empty calorimeters to correct for the asymmetry of the calorimeter is not mathematically sound, but for highest precision must similarly be converted into a heat capacity contribution at zero frequency [15].

\section{Multiple Frequency Modulation}

The final step in the analysis is to eliminate the problem that arises for the use of multiple frequencies from the quickly decreasing amplitudes of the higher harmonics in Equation (5). This was accomplished by replacing a simple sawtooth with one designed to have similar amplitudes for the $1^{\text {st }}, 3^{\text {rd }}, 5^{\text {th }}$, and $7^{\text {th }}$ harmonics. Instead of the standard quasiisothermal sawtooth given by the Fourier series:

$$
T(t)-T_{0}=\frac{8 A_{\mathrm{T}_{3}}}{\pi^{2}}\left[\sin \omega t-\frac{1}{9} \sin 3 \omega t+\frac{1}{25} \sin 5 \omega t-\frac{1}{49} \sin 7 \omega t+\frac{1}{81} \sin 9 \omega t-\ldots\right],
$$

the following complex sawtooth was created [15]:

$$
T(t)-T_{0}=A[0.378 \sin \omega t+0.251 \sin 3 \omega t+0.217 \sin 5 \omega t+0.348 \sin 7 \omega t-0.067 \sin 9 \omega t \cdots] .
$$

Its harmonics are presented in Figure 5 for the sample temperature, $T_{s}$. A single cycle of the complex sawtooth contains 14 segments and can easily be programmed, even for a standard DSC of high quality. It produces simultaneously four modulations with different frequencies and similar amplitudes. If using also the $9^{\text {th }}$ harmonic, which is only increased by a factor of nine, five harmonics can be used to establish $\tau$ with a single measurement and produce high

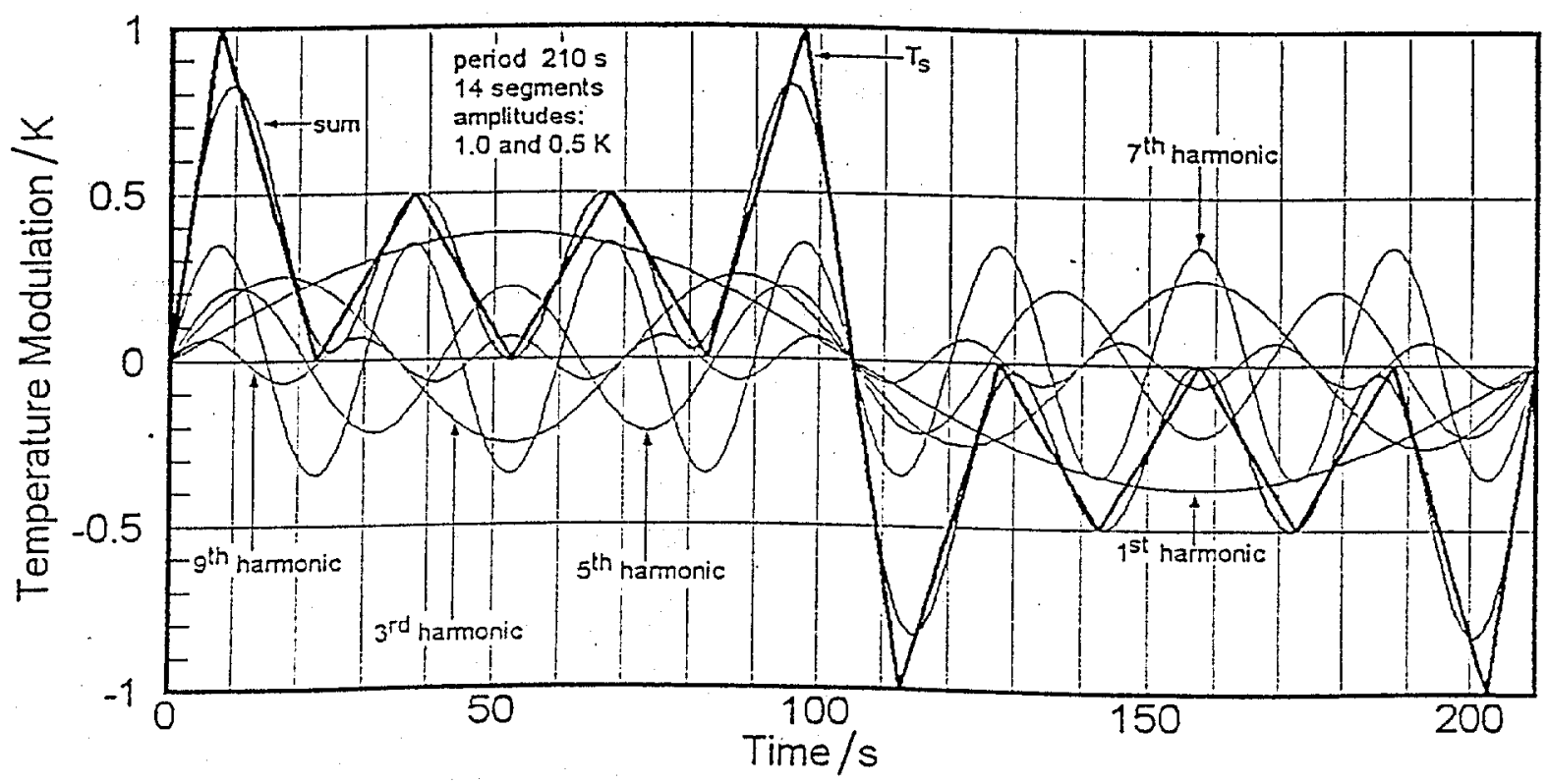

Figure 5-Complex sawtooth modulation showing similar amplitudes of the harmonics. 
quality data for the heat capacity at zero modulation frequency. All heat losses due to drits and losses of other frequency are rejected.

The complex sawtooth was chosen because of the missing even harmonics and the rapid decrease in amplitude of the higher harmonics. The missing harmonics space the measured frequencies more widely, and by summation of the different harmonics, the initial detriment of decreasing amplitudes turns to an advantage since leaving out higher harmonics does not remove much of the measurement after adjusting the first four harmonics. The total temperature variation in Figure 5, shown by the heavy line, is closely fitted by the sum of the first five harmonics (thin line), i.e., adding any higher harmonics cannot yield significant additional information, and all used harmonics have similar contributions (except the smaller $9^{\text {th }}$ harmonic)

\section{Applications}

An application of the complex sawtooth in the measurement of the heat capacity of polystyrene is shown in Figure 6 using the Perkin-Elmer Pyris-1 DSC in a quasi-isothermal mode at $333.6 \mathrm{~K}[16]$. Two overall modulation periods were applied, 210 and $420 \mathrm{~s}$. The heat-flow rates shown in Figure 6 show that during the $15 \mathrm{~s}$ sections of the $210 \mathrm{~s}$ modulation, steady state was not reached (thin curve), while a $420 \mathrm{~s}$ with its $30 \mathrm{~s}$ sections reached steady state for almost half of each modulation section. The sum of the five Fourier components is shown by the thick curves of the figure. The sample temperature recording was only slightly rounded at the changes of heating rate when compared to Figure 5. Analyzing the data of each harmonic with Equation (3) yields the filled symbols in the left graph of Figure 7. Only the harmonics with periods of more than $250 \mathrm{~s}$ give satisfactory data. The dashed line marks

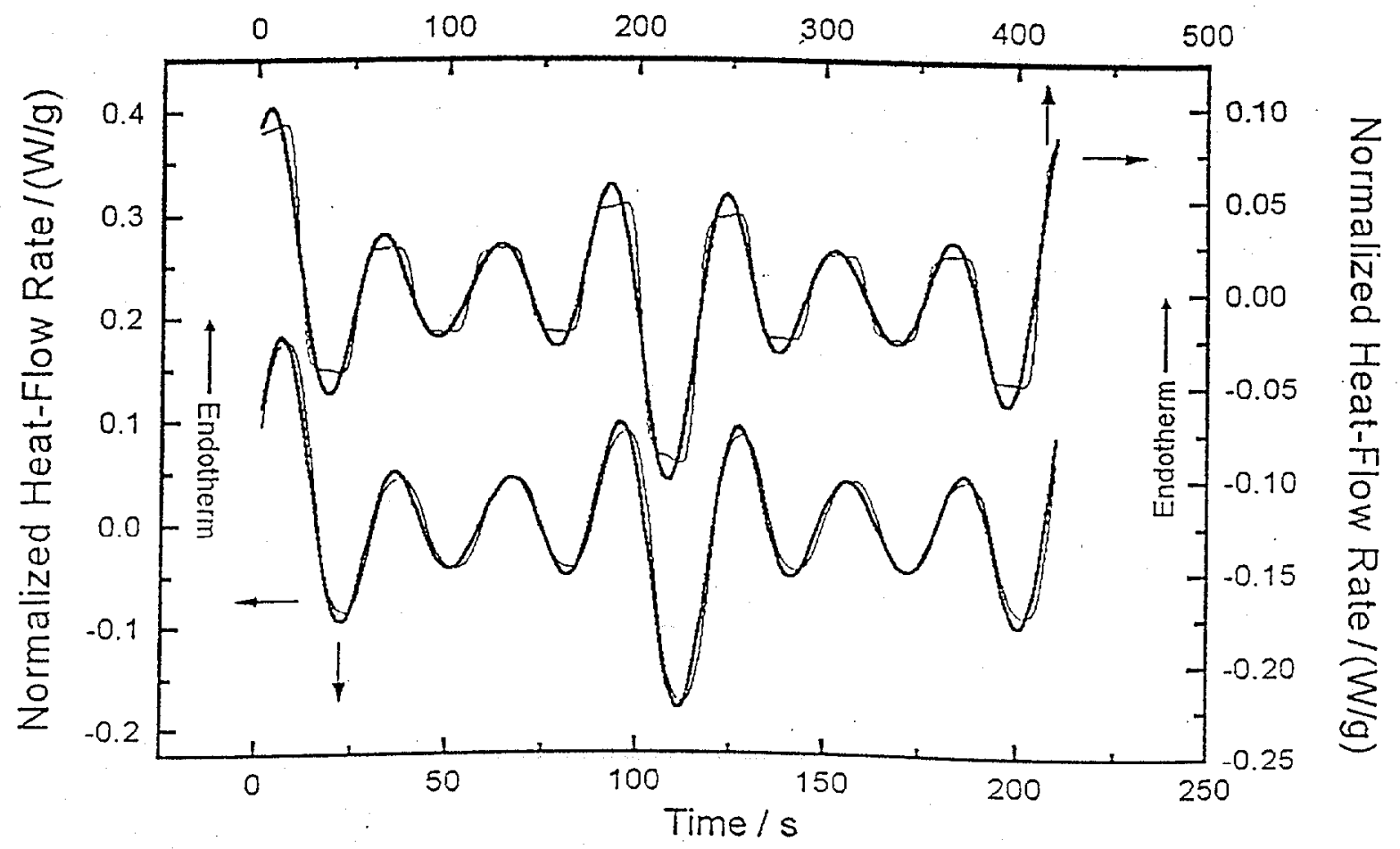

Figure 6 - Complex sawtooth TMDSC (thin: measurement, thick: sum of the harmonics). 

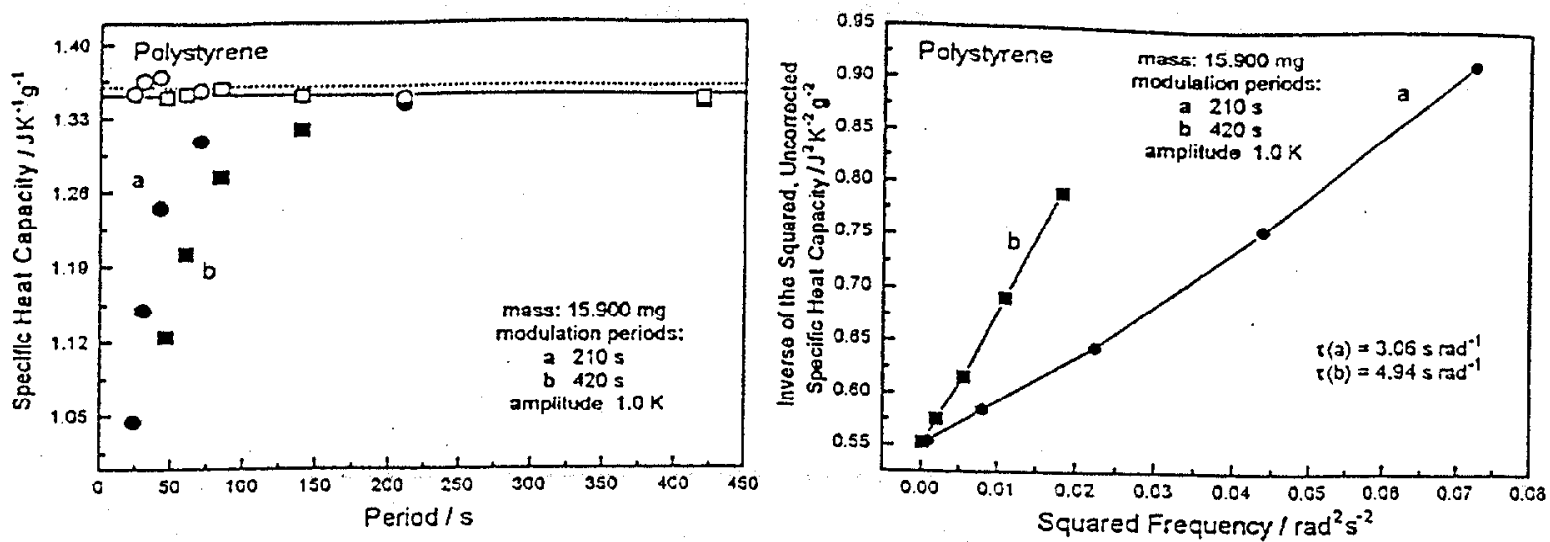

Figure 7 - Analysis of Figure 6 (dashed line literature value, heavy line, average).

the literature value of the polystyrene specific heat capacity, The corrections to Equation (4) are documented in the right graph of Figure 7 . With the indicated values for $\tau$, the open symbol data result, with the heavy line representing the average value $( \pm 0.5 \%)$.

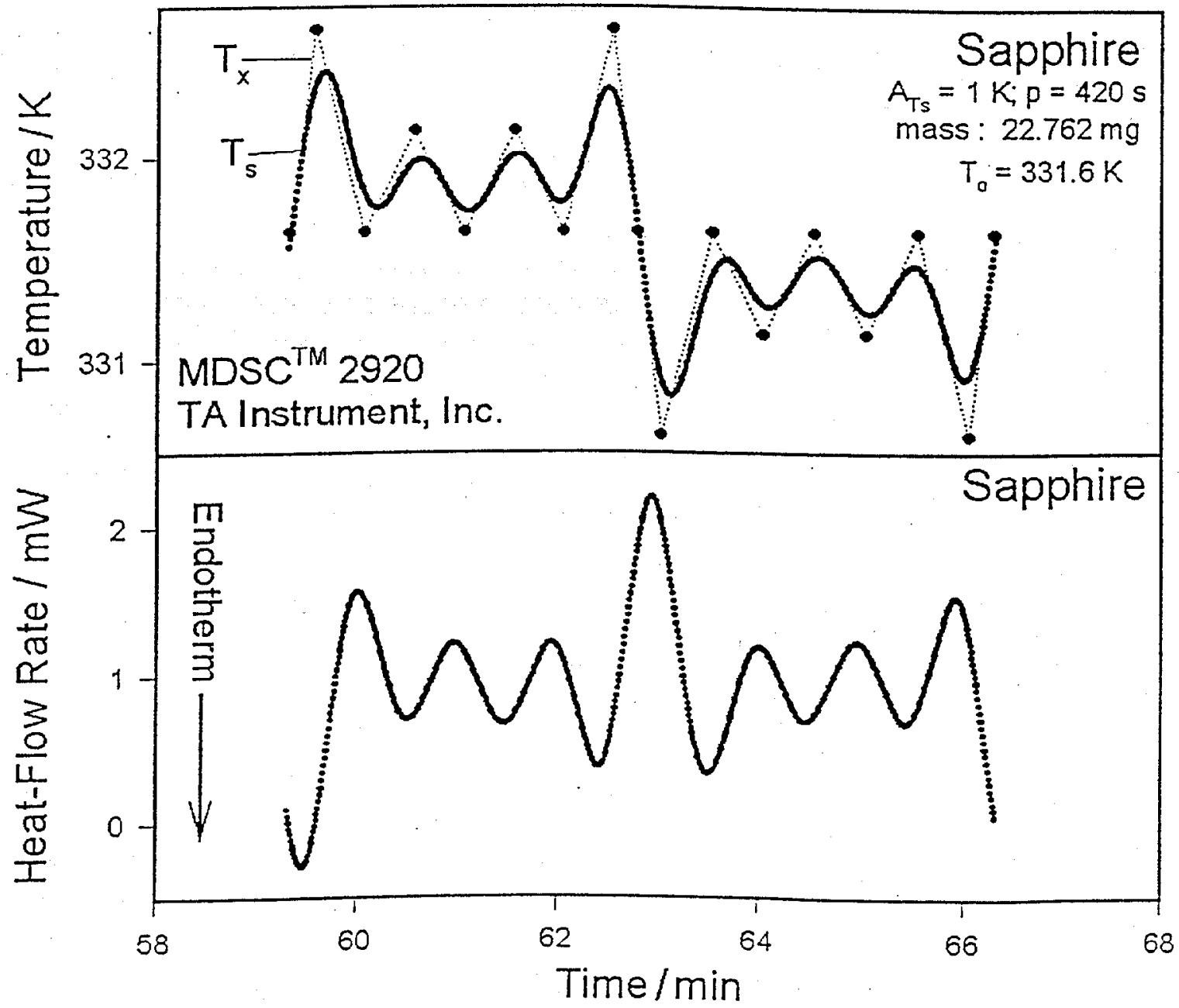

Figure 8 - Temperature-modulation with a complex scwwtooth for a heat-fluc-type DSC. 
Next, data were gathered on sapphire with a heat-flux TMDSC of the TA Instruments type (see Figure 1), programmed with a $420 \mathrm{~s}$ complex sawtooth [17]. Figure 8 shows the sample temperature and heat-flow rate data. Since the temperature sensing occurs far from the heater, the programmed temperature $(\bullet$ ) was not reached, but the $H F$-response remained linear. The heat-flow response does not show any even harmonics, and the $1^{\text {st }}, 3^{\text {rd }}, 5^{\text {th }}, 7^{\text {th }}$, and $9^{\text {th }}$ harmonic represent practically the whole curve of Figure 8 , with amplitudes as expected. The analysis of the data is shown in Figure 9 with an almost constant $\tau$ for all harmonics.

Another heat-flux DSC is the Mettler-Toledo 820. In this calorimeter, temperature is measured close to the heater and used to control the modulation and establish the reference temperature. The temperature difference, however, is established separately with higher resolution, using a heat-flux sensor (FRS-5). Heat capacities were measured quasiisothermally for poly (methyl methacrylate) (PMMA) at $300 \mathrm{~K}$ [18]. Figure 10 illustrates on the left the uncorrected data for the complex sawtooth $(0)$ and also the standard DSC data taken from the different heating $(\Delta)$ and cooling rates $(\nabla)$. The corrected data with a $\tau$ established in the right graph are marked $(\bullet)$. The literature data are given by the solid horizontal. They are closely matched by the experimental data and have a standard deviation of $\pm 0.04 \%$. The average is marked by the dotted line. The literature value is $0.76 \%$ lower than the experimental average, but it must be remembered that the data bank is usually accurate only to within $3 \%$. The graph on the right shows that there is practically no region of constant $\tau$ and a quadratic fit was made with good success, as shown by the drawn-out curve. The heat capacity data were corrected with the frequency-dependent $\tau$.
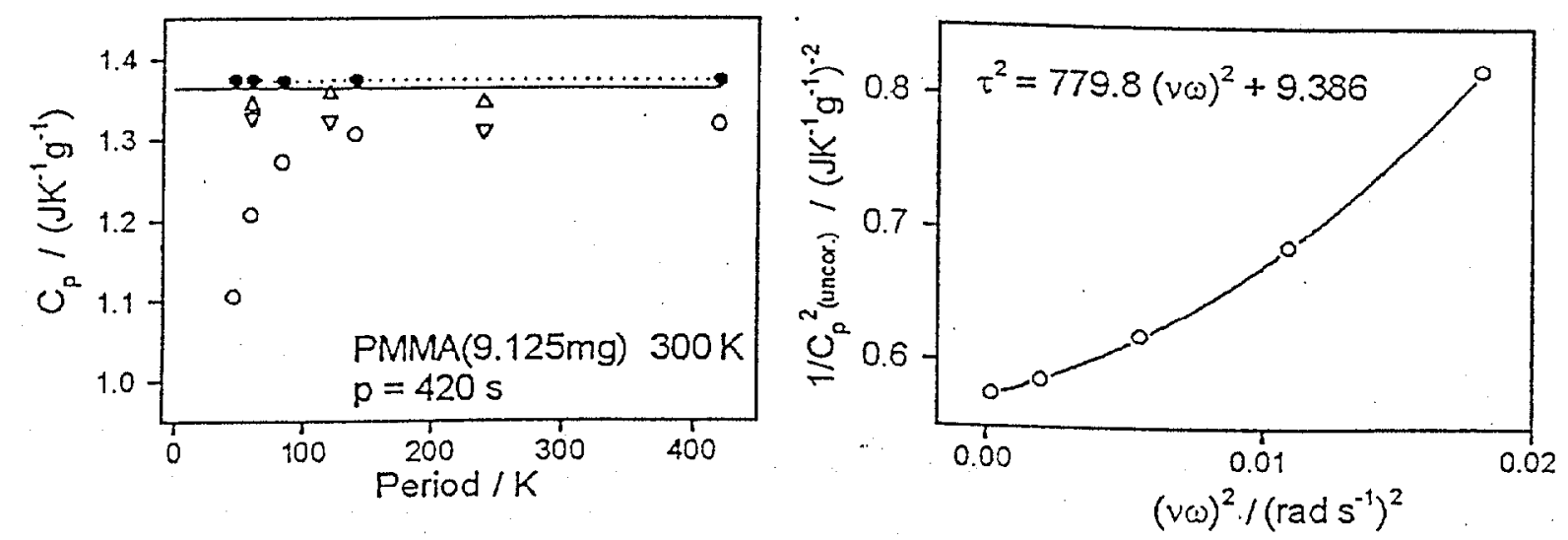

Figure 10 - Analysis of PMMA with a Mettler-Toledo DSC 820.

Also of interest is the Lissajous figure of the complex sawtooth. Figure 11 displays the third to the sixth complex cycle on $22.6 \mathrm{mg}$ sapphire, taken with a modulation period of $420 \mathrm{~s}$ at $300 \mathrm{~K}$. The numerals indicate the sequence of the section of the complex modulation, starting with " 1 " and matching the first half-segment with the last, 15 , which completes the cycle. The width of the lines covers the repeatability.

Besides improving the measurement of heat capacity, which outside of the transition regions react practically instantaneously to a change in temperature, it is of interest to study the slow response of samples in the glass and melting transition regions. The slow response. of the sample adds then to the lags due to the thermal resistances juist discussed. The method of analysis for the additional slow sample response has not been fully developed yet, it must 
be found as a deviation of $\tau$ from the value set by the heat capacity outside the transition region. Using the displayed method, it should then be possible to gain kinetic information on the same sample configuration using a single measurement. A single measurement is of particular importance to have an identical samples for all frequencies.

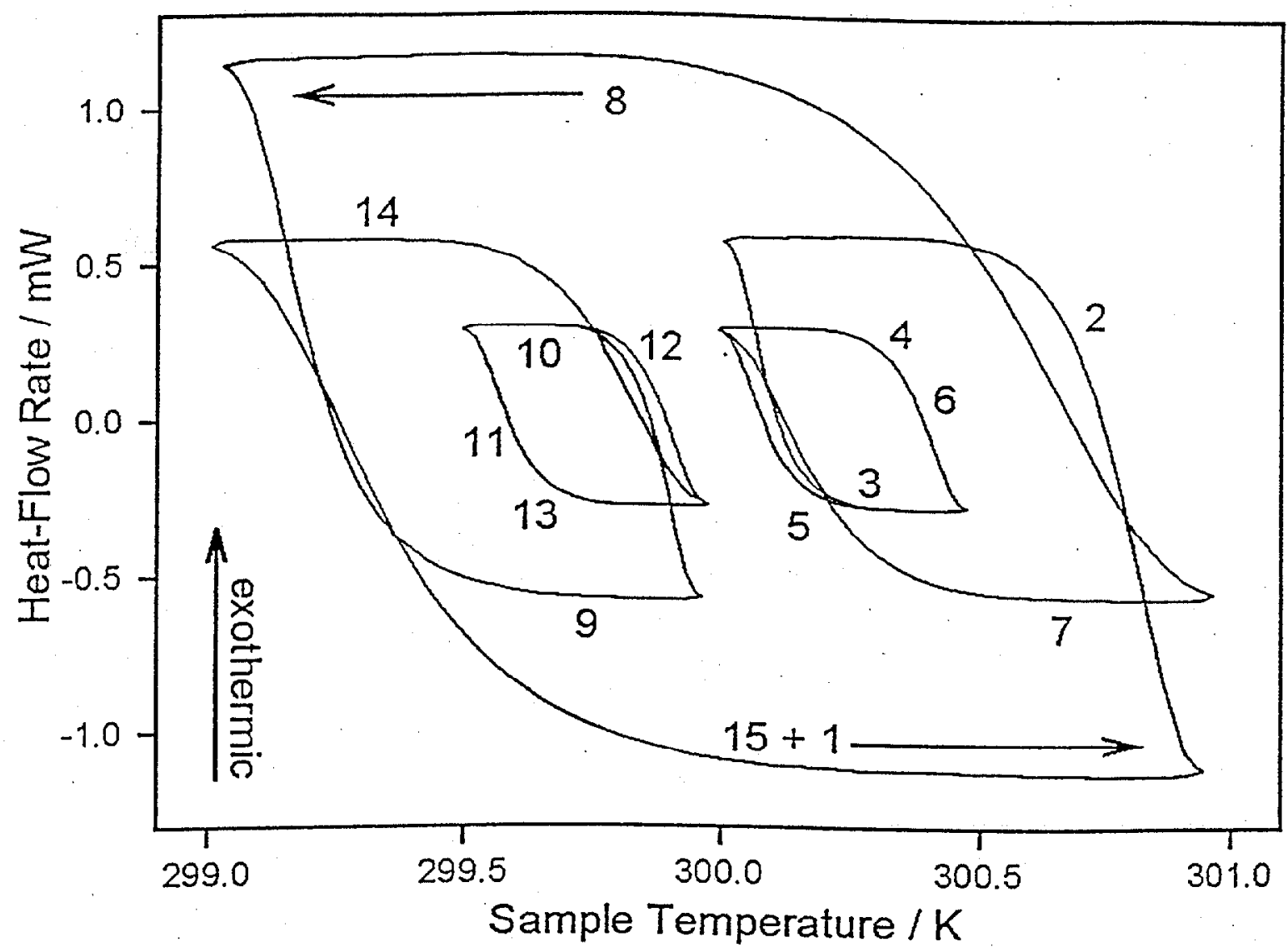

Figure 11 - Lissajous figure of an analysis as Figure 10, but using $22.6 \mathrm{mg}$ of sapphire.

For the glass transition it was found using a single sinusoidal frequency that the sample adds the following effects: (1) higher harmonics due to the cooperative freezing or unfreezing of large-amplitude motion, (2) frequency shifts due to coupling of the linear and modulated time scales if the analysis is not done quasi-isothermally, and (3) asymmetry in the reversing response due to an exponential temperature dependence of relaxation times [19].

The largest differences in the description of the modulated parameter can show up, however, in first-order transitions. The observed effects may be as follows: (1) The transitions may be only partially reversible or even irreversible. (2) Large exotherms or endotherms may cause time periods of constant temperature within the sample and disturb the modulation. (3) Instrument lags due to melting and crystallization may cover more than one modulation period. (4) Irreversible processes may contain accidental Fourier components of the modulation frequency. (5) The transition kinetics may cause lags in the sample response. For recent publications with single-frequency modulation see [20]. In all of these cases it is necessary to inspect the heat-flow rate in the time domain before accepting "apparent heat capacities" computed from a first-harmonic Fourier fit of the reversing parameters. 


\section{Conclusions}

Temperature-modulated calorimetry has experienced recently a strong increase in interest and new methods of modulation and analysis have been developed. The advantage of the multifrequency modulation for the measurement of heat capacity is the possibility to increase the precision by a factor 10 or more over the common $=3 \%$ seen for the standard DSC. This is possible by calibration of the various thermal conductivity effects inside and outside the calorimeters during the actual run by deriving data for different frequencies.

It is proposed that the same method may be able to analyze the frequency-dependence of transition effects by comparison of the calibration factors outside and inside the transition region. This method would for the first time permit a study of the frequency dependence on a sample of identical thermal history for all frequencies.

Since temperature modulation has recently also applied to the analysis of mechanical properties and thermogravimetry, as well as to DSC coupled with atomic force microscopy (microcalorimetry), the here proposed multi-frequency modulation may also be applied to these techniques.

\section{Acknowledgments}

Supported by the Division of Materials Research, NSF, Polymers Program, Grant DMR-9703692 and the Division of Materials Sci., and Engineering, Office of Basic Energy Sciences, DOE at Oak Ridge National Laboratory, managed and operated by UT-Battelle, LLC., under contract number DE-AC05-00OR22725.

\section{References}

[1] M. Reading, M., Hahn, B. K, andCrowe, B. S. U. S. Patent 5,224,775, 6 July 1993.

[2] Sullivan, P. F., and Seidel, Pysical Review, Vol. 173, 1969, p. 679; see also the review by Gmelin, E., Thermochimica Acta, Vol. 304/305, 1997, p. 1.

[3] Gobrecht, H., Hamann, K., and Willers, G., Journal of Physics, E: Scientific Instruments, Vol. 4, 1971, p. 21; Dixon, G. S., Black, S. G., Butler, C. T., and Jain, A. K., Analytical Biochemistry, Vol. 121; 1982, p. 55.

[4] Wunderlich, B., Boller, A., Okazaki, I., Ishikiriyama, K., Chen, W., Pyda, M., Pak, J., Moon, I., and Androsch, R. Thermochim. Acta, Vol. 330, 1999, pg. 21-38.

[5] B. Wunderlich, Thermal Analysis, Academic Press, New York, 1990; updated and expanded as the computer-assisted course Thermal Analysis of Materials, with over 2879 screens, published on the Intemet (web.utk.edurathas/courses/tham $99 . \mathrm{html}$ ), downloadable including presentation software, University of Tennessee Knoxville, Knoxville, TN, 2000.

[6] Wunderlich, B., "Differential Thermal Analysis," in Physical Methods of Chemistry, A. Weissberger and B. W. Rossiter, eds. Vol. 1, Part V, Chapter 8. J. Wiley \& Sons, New York, 1971.

[7] Ozawa, T., Bulletin of the Chemical Society of Japan, Vol. 39, 1966, p. 2071.

[8] Gaur, U. and Wunderlich, B., Journal of Physical and Chemical Reference Data, Vol. 10, 1981, p. 119.

[9] Boller, A. Jin, Y., and Wunderlich, B., Journal of Thermal Analysis, Vol. 42, 1994, 
p. 307.

[10] Wunderlich, B., Bodily, D. M., and Kaplan, M. H., Journal of Applied Physics, Vol. $35,1964,95$.

[11] Androsch, R, Moon, I., Kreitmeier, S., and Wunderlich, B., Thermochimica Acta, in press, 2000.

[12] Hatta, I. and Maramatsu, S., Japanese Journal of Applied Physics, Vol. 35, 1996, p. L858; Hatta, I. and Katayama, N., Journal of Thermal Analysis, Vol. 54, 1998, p. 557.

[13] Höhne, G. W. H., Thermochimica Acta,Vol. 330, 1999, p. 45.

[14] Androsch, R. and Wunderlich, B., Thermochimica Acta, Vol. 333, 1999, p. 27.

[15] Wunderlich, B., Androsch, R., Pyda, M., and Kwon, Y. K., Thermochimica Acta, in press, 2000.

[10] Kwon, Y. K., Androsch, R., Pyda, M., and Wunderlich, B., Thermochimica Acta, submitted, 2000.

[17] Pyda, M., Kwon, Y. K., and Wunderlich, B., Thermochimica Acta, submitted, 2000; Proceedings $27^{\text {h }}$ NATAS Conference, K. R. Williams and K Kociba, edts., Vol. 27 , 1999 , p. 345.

[18] Pak, J. and B. Wunderlich, B., Thermochimica Acta, submitted, 2000; Proceedings $27^{\text {th }}$ NATAS Conference, K. R. Williams and K. Kociba, edts., Vol. 27, 1999, p. 339.

[19] Wunderlich, B. and Okazaki, I., Journal of Thermal Analysis, Vol. 49, 1997, p. 57; Thomas, L. C., Boller, A., Okazaki, I., and Wunderlich, B., Thermochimica Acta, Vol. 291, 1997, p. 85; Okazaki, I. and Wunderlich, B., Journal of Polymer Science, Part B: Polymer Physics, Vol. 34, 1966, p. 2941; Wunderlich, B., Boller, A., Okazaki, I., and Kreitmeier, S., Journal of Thermal Analysis, Vol. 47, 1996, p. 1013; Boller, A., Okazaki, I. and Wunderlich, B., Thermochimimica Acta, Vol 284, 1996, p. 1; Boller, A., Schick, C., and Wunderlich, B., Thermochimica Acta, Vol. 266, 1995 , p. 97.

[20] Androsch, R. and Wunderlich, B., Macromolecules, Vol. 32, 1999, p. 7238; and to be published; see also: Boller, A., Ribeiro, M., and Wunderlich, B., Journal of Thermal Analysis and Calorimetry, Vol. 54, 1998, p. 545; Ishikiriyama, K. and Wunderlich, B., Macromolecules, Vol. 30, 1997, p. 4126; and Journal of Polymer Science, Part B, Polymer Physics., Vol. 35, 1997, p. 1877; Okazaki, I. and Wunderlich, B., Macromolecular Chemistry and Physics, Rapid Communications, Vol. 18, 1997, p. 313; and Macromolecules, Vol. 30, 1997, 1758. 